\title{
Grief's impact on sensorimotor expectations: an account of non-veridical bereavement experiences
}

\author{
Becky Millar ${ }^{1} \mathbb{D}$
}

Accepted: 15 July 2021 / Published online: 11 August 2021

(C) The Author(s) 2021

\begin{abstract}
The philosophy of grief has directed little attention to bereavement's impact on perceptual experience. However, misperceptions, hallucinations and other anomalous experiences are strikingly common following the death of a loved one. Such experiences range from misperceiving a stranger to be the deceased, to phantom sights, sounds and smells, to nebulous quasi-sensory experiences of the loved one's presence. This paper draws upon the enactive sensorimotor theory of perception to offer a phenomenologically sensitive and empirically informed account of these experiences. It argues that they can be understood as deriving from disruption to both sensorimotor expectations and perceived opportunities for action, stemming from the upheaval of bereavement. Different facets of the enactive sensorimotor approach can help to explain different types of postbereavement perceptual experience. Post-bereavement misperceptions can be accounted for through the way that alterations to sensorimotor expectations can result in atypical 'amodal completion', while bereavement hallucinations can be understood as 'appearances' that fail to form part of the usual patterns of sensorimotor contingency. Quasi-sensory experiences of the presence of the deceased can be understood as resulting from changes to perceived affordances. This paper aims to demonstrate the explanatory value of key aspects of the sensorimotor approach by highlighting how they can help to explain the phenomenology of post-bereavement experiences. However, it also illuminates certain areas in which the sensorimotor approach ought to be supplemented, especially if it is to account for tight connections between perception, affect, and intersubjectivity that are salient in grief.
\end{abstract}

Keywords Grief · Bereavement $\cdot$ Hallucinations $\cdot$ Sensorimotor enactivism

Becky Millar

becky.millar@york.ac.uk

1 Department of Philosophy, University of York, York, UK 


\section{Introduction}

Grief involves profound emotional upheaval, but it also has wide-reaching implications for other aspects of human experience, many of which have received comparatively little philosophical attention. In particular, misperceptions, hallucinations and other anomalous perceptual experiences are strikingly common following a bereavement. This paper offers an account of these non-veridical and unusual post-bereavement experiences. Drawing upon the enactive sensorimotor theory of perception, I propose that many such experiences result from disruption to sensorimotor expectations and perceived opportunities for action. When a loved one dies, one's implicit expectations related to the loved one's presence are violated, and the sensory implications of one's own worldly engagement are thus likewise altered. The suggested approach offers an account of non-veridical experiences that often occur during grief that is accurate regarding their phenomenology and informed by empirical research surrounding both grief and perception.

The sensorimotor theory of perception (e.g., Hurley, 1998; O'Regan \& Noë, 2001; Noë, 2004) has offered a promising account of perceptual experience, helping to explain and generate predictions with regards wide-ranging and puzzling perceptual phenomena. The approach tells us that the phenomenal character of perceptual experiences is explained by the perceiver's implicit, practical expectations regarding sensorimotor contingencies - law-like relations between motor action and sensory changes. For example, we perceive a largely stable, coherent world before us despite dramatic and continuous shifts in visual stimulation as our eyes saccade. This ability to perceive coherent, invariant objects and scenes is taken to be (at least partly) due to expectations regarding the sensory implications of one's own body movements. A perceiver's ability to fluently engage with the world is said to be laden with these implicit expectations. In recent years, researchers have extended such an approach to other domains, such as social engagement and empathy (Chemero, 2016) and atypical experiences like post-traumatic stress disorder (Ataria, 2015) and psychopathology more generally (Myin et al., 2015). This paper argues that the approach also enables us to better understand perceptual facets of grief ${ }^{1}$ through consideration of how bereavement involves disruption to relevant patterns of sensorimotor contingency.

\footnotetext{
${ }^{1}$ This paper will not address in any detail the issue of whether the perceptual experiences that can occur in reaction to bereavement should truly be understand as part of grief, or whether they are better understand as a reaction to, or frequent accompaniment to, grief, and the argument presented doesn't hinge upon a particular answer to this question. However, it is worth noting that grief is often said not to be a mental state or event, but rather a complex process composed of heterogenous ingredients (e.g., Goldie, 2011), and there seems little reason to automatically exclude perceptual experiences from grief's complex pattern. Moreover, other researchers argue that grief is characterised by a tension between experiences of presence, experiences of absence, and experiences of presence in absence (e.g., Fuchs, 2018, p. 51 describes this as grief's 'core conflict'), which is suggestive of grief having an important perceptual dimension.
} 
Regardless of whether one endorses the sensorimotor approach wholesale, as we shall see, key concepts and strategies drawn from the approach can elucidate the kinds of perceptual experiences that occur amid the disruption to one's world caused by a bereavement. This paper's purpose is therefore not to defend sensorimotor enactivism, and its arguments are likely compatible with different but complementary approaches that also emphasise the role of expectations regarding the sensory upshots of motor action. ${ }^{2}$ Rather, the paper's aim is to demonstrate the explanatory potential of such an approach, particularly with regards its accounts of sensorimotor expectations, affordances, and the problem of 'perceptual presence' - our ability to perceive whole objects despite the limitations of our sensory contact with the world. These aspects of the sensorimotor approach can, I argue, allow for an empirically informed and phenomenologically sensitive account of key types of anomalous post-bereavement perceptual phenomena. Although the focus here is bereavement experiences, the approach may also have wider applicability.

The paper proceeds as follows. Section 2 introduces a rough taxonomy of non-veridical experiences that often follow bereavement. Section 3 discusses the role of sensorimotor expectations in perceptual experience and how sensorimotor disruption can impact perceptual phenomenology. I argue that sensorimotor expectations are contravened in important ways by the absence of a loved one. Section 4 sets out how the sensorimotor approach can offer an account of postbereavement non-veridical experiences that accurately captures the associated phenomenology. First, our violated expectations can allow for atypical instances of 'amodal completion', accounting for misperceptions common in bereavement. Second, the approach allows for non-veridical 'appearances', which are not successfully integrated into our understanding of sensorimotor contingencies. This helps to account for hallucinatory bereavement experiences and explains why such experiences frequently lack the 'perceptual presence' characteristic of objective perception of the world. Third, bereavement disrupts the opportunities for action that one perceives, helping to account for nebulous quasi-sensory experiences of presence. Section 5 highlights certain limitations of the sensorimotor approach in accounting for post-bereavement experiences and suggests some avenues for supplementing the approach. Section 6 discusses some further implications of the approach set out in this paper.

\footnotetext{
${ }^{2}$ For example, similar claims could be made through the lens of autopoietic enactivism (Varela, Thompson, \& Rosch, 1991; Thompson, 2007). This paper draws upon the sensorimotor variant of enactivism due to its specific focus on sensorimotor expectations and sensorimotor disruption and will not discuss the potential overlap and differences between these approaches. Various authors have also taken the sensorimotor approach to be compatible with the increasingly influential predictive processing framework, which offers a plausible story about the mechanistic underpinnings of our sensorimotor expectations (see, e.g., Seth, 2014; Kirchhoff \& Kiverstein, 2019). According to such an approach, our sensorimotor expectations are implemented through counterfactually rich generative models, whereby the brain generates predictions not only with regards the sensory states that will occur but also those that would counterfactually occur on the basis of possible actions (Seth, 2014).
} 


\section{Taxonomising non-veridical bereavement experiences}

Non-veridical perceptual experiences often occur after bereavement. Studies report that between 30 and $60 \%$ of bereaved spouses have experienced so called 'postbereavement hallucinations' (e.g., Rees, 1971, 1975; Castelnovo et al., 2015). ${ }^{3}$ These experiences may not be maladaptive, with many reporting them to be comforting (Parkes, 1970), and some researchers take such experiences to be normal expressions of our 'continuing bonds' with the deceased (e.g., Klass et al., 1996; Steffen $\&$ Coyle, 2011). Despite the strikingly high percentage of people who undergo these experiences, these phenomena remain poorly understood. In particular, the nature of what have been termed 'post-bereavement hallucinations' appear to be heterogeneous, but this is often obscured by researchers failing to distinguish them, using terms like 'hallucinations', 'illusions' and 'experiences of presence' interchangeably, or using different terminology to describe the same phenomena. For example, some studies use the term 'illusion' to refer to non- or quasi- sensory experiences of presence, but do not distinguish hallucinations and misperceptions (e.g., Rees, 1971). Thus, I shall begin by offering a rough taxonomy of post-bereavement experiences.

Non-veridical post-bereavement experiences fall into three main categories: misperceptions, sensory hallucinations, and quasi-sensory experiences of presence. First, cases of misperception (or illusion) following a bereavement are very common (Clayton, 2000; Parkes \& Prigerson, 2010, pp. 58-59; Castelnovo et al., 2015). It is likely that many experiences that have been termed bereavement 'hallucinations' in fact fall into this category. Existing sights, sounds and smells are misperceived as being related to the deceased. One may misperceive a stranger in a crowd as the deceased or misinterpret a creak on the stairs as being their footsteps (Parkes, 1970; Clayton, 2000). ${ }^{4}$ Where one misperceives a person or object as being associated with the deceased, the experience is often transient, being quickly rectified by closer attention to the object in question. Such cases are generally taken to result from the bereaved having a 'perceptual set' (i.e., perceptual expectations or biases) for the deceased (Parkes, 1970; Parkes \& Prigerson, 2010). As we shall see in 4.1, the sensorimotor approach can concur with the emphasis on perceptual expectations here, and offer further clarification of the manner in which expectations allow for such misperceptions.

\footnotetext{
3 The vast majority of the research into non-veridical bereavement experiences has focused upon spousal bereavement. Therefore, the extent to which other forms of bereavement are likely to trigger such experiences is unclear. With the approach to be set out in this paper, we should expect such experiences to be especially common in cases where one's life is closely entwined with that of the person who died, since in such cases one is likely to have a greater suite of expectations regarding the presence of the deceased. However, it is plausible that in other cases, one will still have a more limited set of expectations regarding the presence of the deceased, which could manifest in terms of non-veridical experiences in more constrained circumstances. For example, one might have powerful expectations about seeing their deceased grandma when visiting her former home.

${ }^{4}$ Certain cases, such as misperceiving a creak, might raise questions about whether one's perceptual experiences have gone awry or whether it is only one's post-perceptual judgments that have gone wrong. In Sect. 3.1 I shall say a little more about why, with a sensorimotor approach, one is likely to construe this as a perceptual phenomenon.
} 
Secondly, full-blown hallucinations are cases where one has an experience as of some worldly object, but which is not a genuine perception of anything in the subject's environment. This is distinguished from illusions or misperceptions: cases where an object is perceived, but perceptually appears to have properties it does not have. It is unclear how prevalent full-blown hallucinations are in the context of bereavement, but some describe post-bereavement experiences that are not easily understood as misperceptions. For example, Parkes (1970, p. 194) discusses subjects seeing their deceased loved ones in ways that 'were clearly unrelated to pre-existing sensory stimuli'. One reported seeing her deceased husband gardening while wearing only his trousers, and another reported seeing her father standing beside her bed at night. ${ }^{5}$ Although such cases may not always be clearly distinguishable from misperceptions and other experiences such as dreams, the sensorimotor approach can accommodate the possibility of hallucinatory experiences that differ from both and offers an explanation of the phenomenal character of these experiences (see Sect. 4.2).

Finally, the most commonly reported non-veridical post-bereavement experience is the quasi-sensory feeling of the 'presence' of the deceased (Grimby, 1993; Castelnovo et al., 2015; see Ratcliffe, 2020b for a detailed phenomenological account of such experiences), which are generally not taken to involve any specific sensory modality. Steffen and Coyle (2011 p. 589) characterise the experience as one where, 'There is a sense that the deceased loved one is temporarily present in an almost physical form, perceived partly through near-sensory awareness and partly through an [...] intuitive knowing.' These experiences often involve the feeling that the deceased is nearby in some unspecified location, although they are not seen or heard. I shall discuss such experiences in Sect. 4.3, highlighting how they can be understood in terms of affordances - perceptible opportunities for action.

These different types of non-veridical encounter with the dead are only fuzzily distinguished. There are grey areas regarding whether an experience of presence involves a sensory component or not, and whether a particularly radical misinterpretation of the external world constitutes a hallucination or merely a misperception. Dreams and imagination provide further complications that will not be addressed here. Nevertheless, these different kinds of non-veridical experience appear to have differing traits. For example, they seem to have different temporal trajectories. Grimby's (1993) study of bereavement among the elderly reports that (what they refer to as) post-bereavement hallucinations decline considerably over the first year of the bereavement, whereas experiences of the presence of the deceased did not. There is also some research suggesting that 'externalised' expressions of one's continuing bonds with the dead - i.e., sensory illusions and hallucinations - are associated with violent death and feelings of responsibility for the bereavement, while 'internalised' expressions of continuing bonds (such as evoking an image of the deceased as a comforting presence) are positively associated with personal growth (Field \& Filanosky, 2009).

\footnotetext{
${ }^{5}$ See also Sacks (2012) for discussion and examples of bereavement hallucinations.
} 


\section{Sensorimotor theory: expectations and disruption}

According to the enactive sensorimotor approach, the phenomenology of perceptual experience outstrips current sensory contact with the world. Thus, a core explanandum of the approach is what has been referred to as the problem of 'perceptual presence' (otherwise referred to as amodal completion) - our ability to perceive whole objects and scenes despite the limitations of our current sensory contact with the world. It is said to be through sensorimotor expectations - implicit expectations regarding the counterfactual sensory effects of one's engagement with the world - that we are able to experience whole meaningful objects and detailed scenes. These expectations involve an understanding of how to access other parts of the objects through moving our own bodies or the objects in question. As we shall see in Sect. 4.1, this allows for an explanation of an important type of non-veridical experience, when our sensorimotor expectations go awry.

The approach is importantly action-oriented, generally endorsing the Gibsonian idea that we perceive 'affordances' - opportunities for action. For Noë (2004, p. 105), 'To perceive is (among other things) to learn how the environment structures one's possibilities for movement and so it is, thereby, to experience possibilities of movement and action afforded by the environment.' One's expectation-laden engagement with an object requires a grasp of at least some of the ways this object can be interacted with. Without this, one would not be able to form expectancies regarding the sensory upshots of different possible kinds of interaction. Objects are thus said to afford an exploratory profile, enabling the perception of whole, voluminous objects (see Roberts, 2009). It is natural for the sensorimotor theorist to further acknowledge that we perceive larger-scale opportunities for action, which helps explain how skilful perceivers can experience objects as meaningful, thereby enabling fluent engagement with them. For example, seeing a chair as a chair plausibly requires not only a grasp of how to bring other parts of the chair into view, but also the perception of the chair as affording sitting.

The sensorimotor approach takes much of its support from examples of sensorimotor disruption and adaptation. For example, the sensorimotor approach provides an explanation of evidence regarding vision inverting or displacing goggles (e.g., Hurley, 1998; O’Regan \& Noë, 2001; Hurley \& Noë, 2003; Degenaar, 2013; Ward, 2016). These goggles disrupt the usual sensorimotor contingencies involved in perception but users gradually adapt to novel patterns of sensorimotor contingency, allowing their perceptual experience to revert to some kind of normality. While wearing vision displacing goggles, movement initially causes an array of confusing sensory effects and distortions (Kohler, 1962, 1964; Taylor, 1962), and proprioceptive feelings of movement conflict with visual experience. Use of these goggles can cause strange and hard-to-pin-down phenomenological upshots. Kohler describes subjects who feel they are in a 'topsy-turvy world' where objects appear rubbery and distorted and people seem 'jellylike' (1964, p. 64). Taylor (1962) reports that after some time wearing the goggles, in addition 
to seeing an object in the reversed position, the subject begins to see a ghostly object in the correct location. Such cases highlight how sensorimotor disruption can lead to odd, anomalous sensory encounters, which as we shall see, occurs in cases of grief also.

Taking sensorimotor disruption to explain why certain distressing life experiences result in unusual perceptual effects has a precedent in the literature surrounding sensorimotor theory. Ataria (2015) proposes such an approach can allow us to better understand the perceptual effects of trauma and others have argued that a sensorimotor approach can aid our understanding of psychopathology more generally (Myin et al., 2015). Ataria's account of post-traumatic stress highlights the ways that disruption of one's practical know-how during trauma experiences, particularly in relation to the 'freeze' response, can affect and impair perceptual abilities. According to Ataria (2015), when one is in a state of freeze response, one's practical capacities are impaired and may even collapse entirely, which can explain why trauma can, in extreme cases, result in temporary blindness or deafness:

When the ability to predict based on a set of (implicit) sensorimotor laws declines, one's ability to perceive may also be damaged. Indeed, when the sensorimotor loop weakens, the ability to predict is reduced; one's practical knowledge becomes weaker and in radical cases may even collapse. (p. 147)

The next section argues that grief too involves disruption of our usual sensorimotor skills. The loss of a loved one can result in disruption to sensorimotor engagement due to one's expectations regarding the presence of the deceased being thwarted.

\subsection{Post-bereavement sensorimotor disruption}

Grief involves disruption to almost every aspect of one's life. Parkes (1988) argues that grief involves major alterations to one's 'assumptive world' - one's taken-forgranted system of beliefs, which allows them to fluently act and plan. Attig (1996) makes the related claim that grief is a process of relearning one's world, encompassing emotional, psychological, behavioural, spiritual and social, physical and biological elements. Maclaren (2011, p. 62) tells us that the 'genuine passion' of grief 'consists in a breakdown of our habitual negotiation of the world'. Ratcliffe (2017) similarly takes grief to be characterised by a rupture to 'wide-ranging system of habitual expectations and practical meanings' (p. 166). Here I focus on disruption to perceptual skills and how this results in anomalous perceptual experiences (although, as we shall see in Sect. 5, these capacities are intimately connected to broader aspects of lived experience). I argue that the loss of a loved one can result in significant violations of one's sensorimotor expectations, thus offering an explanation of the non-veridical and confusing perceptual experiences that often occur following a bereavement.

The sensorimotor approach does a good job of explaining various types of perceptual distortion and anomalous perceptual experiences in terms of sensorimotor disruption. A key way that sensorimotor disruption can occur for the bereaved is 
through the violation of one's implicit expectations related to seeing, hearing, touching and otherwise engaging with a person who has died. An initial way in which post-bereavement disruption of this sort can be evidenced is through research showing that early stages of mourning are characterised by 'search' efforts to recover the deceased (Parkes, 1970, 1996; Bowlby, 1980), indicating that in some sense the bereaved 'expect' the deceased to show up. In a wide range of contexts, bereaved subjects have sensorimotor expectations specific to their deceased loved one, and this shows up in such search activities, from scanning the environment for any sign of the deceased to reaching out for one's deceased spouse while lying in bed. The violation of the expected sensory upshots of one's search efforts constitutes a kind of sensorimotor disruption, which can plausibly have the kinds of perceptual upshots to be discussed in Sect. 4. The absence of the deceased can disrupt one's expectation-laden sensorimotor engagement to such an extent that empirical work has found likenesses between bereavement and sensory deprivation. Hofer (1984) finds that the 'loss of customary environmental stimulation' associated with sensory deprivation 'seems to reproduce several of the cardinal symptoms of the bereaved' (p. 189). Both grief and sensory deprivation can produce perceptual distortions, as well as disruption to our overall mental and physical functioning. Hofer claims we are dependent on the patterns and levels of sensory stimulation in regulating our mental and physical functioning. When such levels are not sufficient or the patterns of stimulation are radically changed, this can result in impairments of our capacities.

$[\mathrm{W}] \mathrm{e}$ notice that our concentration and attention are impaired, our perception is somewhat distorted, we do not sleep well, our appetite is reduced or subject to sudden cravings, and we are periodically overcome with fatigue. In its extreme form, we feel that we are falling apart mentally. We see and hear things that are not there. (p. 191)

Although according to Hofer, bereavement does not involve the level of sensory change that sensory deprivation does, where one person is of particularly great importance to another, 'the loss of this one source may actually constitute a massive deprivation of relevant environmental input' (p. 189). ${ }^{6}$ Certain patterns of environmental stimulation may be crucial for one's functioning, and these patterns may involve a specific other person.

Although sensorimotor expectations are often appealed to in explanations of the perception of straightforward objects, such as tomatoes with hidden backs, they are also used by sensorimotor theorists in accounting for more complex perceptual phenomena. For example, Noë (2006) highlights that such expectations might allow for the experience of experimental music as something other than 'mere noise' ( $p$. 31). One must have a degree of understanding of how the melody will unfold for the music to make sense. Thus, sensorimotor expectations are taken to be interwoven and continuous with one's broader understanding of, and expectations about, the world. Against such a backdrop, it is reasonable to allow for one's sensorimotor

\footnotetext{
${ }^{6}$ See also Guenther (2013) for further discussion of the links between sensory deprivation and social deprivation.
} 
expectations to also relate to specific individuals. One may have expectations that moving towards the kitchen will bring a loved one into view or that further investigation of a creaking sound would reveal it to be the sound of a loved one's footsteps.

Because of the continuity between sensorimotor expectations and one's broader understanding of the world, the line between perception and judgment is somewhat blurred, and so the line between perceptual illusions and false judgements is likely to be somewhat blurred too. ${ }^{7}$ However, the sensorimotor theorist is likely to reject the attribution of experiences like hearing a sound as a loved one's footsteps to merely post-perceptual judgment rather than to perception itself. Such experiences are taken to involve the immediate perception of action opportunities or affordances. The affordances presented by an experience as of a loved one's footsteps will differ from those presented by an experience as of a creak. To the extent that affordances are understood as aspects of perceptual experience, an experience as of a loved one's footsteps, will be importantly perceptual too.

The blurred line between sensorimotor expectations and more explicit types of expectation and thought should not be taken to imply that they are inseparable. Despite this continuity and the fact that sensorimotor expectations are intended to allow for meaningful perception of potentially complex phenomena, sensorimotor expectations are themselves generally taken to be implicit and practical. The term 'expectation' might be suggestive of propositional or explicit thought, but as Myin, O'Regan, \& Myin-Germeys note:

There is no logical need for this, as an expectation can exist as a sensitivity or adaptation to a particular context. An animal can be on alert when nearing a particular place because, based on its own history, it expects the presence of another animal at that place. But nothing logically requires that such sensitivity should only be explicable by a content-carrying inner episode, let alone one involving a proposition. (2015, p. 362)

Thus, sensorimotor expectations are generally taken to be a kind of implicit, nonpropositional know-how. If this is the case, one's sensorimotor expectations can come apart from one's explicit judgements. For example, one may know that their loved one has died, and yet still in some sense expect the deceased to be present, as indicated by, for example, 'search' behaviours (Parkes, 1996). One's implicit expectations relating to the deceased - despite explicit judgements that the person has died - can shape their perceptual encounters with the world.

The claim that bereavement involves the violation of sensorimotor expectations relates to complementary research highlighting the important ways in which one is habituated towards the presence of others (e.g., Fuchs, 2018; Køster, 2020, 2021; Ratcliffe, 2020a, 2020b) and the role that this plays in bereavement phenomenology. Køster $(2020,2021)$ outlines how one's bodily memories of the deceased play a role in the sensing the continued presence of the deceased. These embodied memories,

\footnotetext{
7 See Noë (2009) for discussion of the continuity of perception and thought within his version of the sensorimotor approach. Thought is taken to be another means of access to the world, and the approach permits of borderline cases between thought and straightforwardly perceptual access.
} 
he claims, involve one's habituation towards the 'felt sense of the concrete other' and encompass auditory, olfactory and gustatory, visual, tactile, and kinaesthetic elements. He argues it is through this habituation that people are able to experience 'continuing bonds' with the dead. Fuchs relatedly states, 'This felt presence is the result of an accumulated history of encounters and interactions with the loved one that has left its traces in one's lived body and lived space' (2018, p. 47). The sensorimotor theorist can concur with such claims about how we become attuned to the presence of another. With this approach, such habituation can be construed as elements of our sensorimotor skills - we have expectations regarding our possibilities for interacting with another person and the sensory upshots of this interaction.

\section{Non-veridical bereavement experiences within a sensorimotor framework}

We have seen there is good reason to think that bereavement involves sensorimotor disruption. However, I have not yet addressed in detail the phenomenological implications of such a disruption and whether they can account for reported nonveridical post-bereavement experiences. Here I shall outline how the three types of non-veridical bereavement phenomena that were roughly distinguished in Sect. 2 can be explained by disruption to sensorimotor skills. As we shall see, the enactive sensorimotor approach can provide an account of post-bereavement illusions and hallucinations that is illuminating and faithful to the phenomenology of these nonveridical experiences. It will become clear that different facets of the approach can explain different types of non-veridical bereavement experience.

\subsection{Atypical amodal completion and misperception}

As noted in Sect. 3, sensorimotor enactivism can explain aspects of perception that would - under certain common assumptions about perceptual experience - be puzzling. One such feature of perceptual experience is the ability to perceive whole objects and scenes despite limitations of sensory contact with the world, i.e., the puzzle of perceptual presence or amodal completion. That the sensorimotor approach dissolves this puzzle is taken to be one of its key virtues, and although there are many other possible explanations of the phenomenon of perceptual presence or amodal completion, Noë $(2002,2004)$ argues that only the enactive sensorimotor account is accurate with regards the associated phenomenology. One's perceptions of whole objects and detailed scenes involve a tension that is evident upon phenomenological reflection: they involve both an experience associated with a limited sensory perspective (i.e., in one sense, we only see the facing side of the tomato) but, in another sense, we experience the whole. For the sensorimotor theorist, this tension is explained by our tacit expectations about how movements of one's body (or of the object in question) would allow sensory access to further aspects of the object, giving rise to the experience of the whole or 'gestalt'. One's experience of the presence of wholes is thus said to be 'virtual' (Noë, 2004, pp. 49-52) in that it is based upon 
our understanding of how to access the hidden parts. This solution is taken to reflect the phenomenology of our experiences (thereby providing evidence for the theory) and render them unpuzzling. It is said that upon close phenomenological reflection, perceptual experience is precisely as the sensorimotor account describes: we perceive a world that is available to us in all its detail, rather than it seeming as though we have immediate awareness of all this detail.

The way the sensorimotor approach allows the dissolution of the puzzle of perceptual presence also has the advantage of offering a phenomenologically plausible explanation of important types of non-veridical experience, further highlighting the approach's explanatory value. The approach, I argue, renders both amodal completion and consequent misperceptions unpuzzling, and is particularly well-suited to explaining a very common type of post-bereavement misperception.

Where sensorimotor expectations are violated, the experience of the whole (i.e., three-dimensional objects and detailed scenes) may be atypical or non-veridical. Because perception is taken to be underdetermined by current sensory underpinnings, and particularly where there is not much temporally extended exploration of the environment, there is scope for us to perceive objects in atypical ways. For example, where we take ourselves to experience a whole voluminous tomato, but in fact it is a tomato façade, our sensorimotor expectations have been breached in some sense (Hurley \& Noë, 2006). And the experience as of a whole tomato is nonveridical. Reaching over to grasp the tomato but being met with only a façade is likely to correct this non-veridical experience and will also likely give to a somewhat disconcerting experience of the absence of the expected spherical object. In such cases, upon reflection we can notice that important details (such as the back of the tomato) were in fact lacking in our immediate sensory awareness. Although there is a sense in which we perceive the whole, we can also notice the other important sense in which we do not perceive the hidden elements of objects and scenes in all their detail. Thus, although one may find their misinterpretation of an object or scene surprising, the surprise is not due to those features with which one has immediate sensory contact being experienced non-veridically. Rather, it is one's sensorimotor expectations about how to access further details that have gone awry.

Grief can result in non-veridical and atypical interpretations of objects and scenes in a way that accords with one's tacit expectations, ${ }^{8}$ and again this is manifest upon phenomenological reflection. This account of misperceptions is especially wellsuited to explain common short-lived non-veridical experiences associated with grief. An extremely prevalent type of post-bereavement experience is that of misperceiving a stranger in a crowd as being the deceased (Clayton, 2000, p. 306). For a moment, the bereaved recognises this person as being their loved one, and yet with closer attention, it becomes clear that it is a stranger with only a passing resemblance to the person who died. By bringing the details of a person's face into view, the misperception is corrected. One may experience a disconcerting clash between

\footnotetext{
${ }^{8}$ As discussed in Sect. 3.1, one piece of evidence for such expectations being a prevalent feature of grief is provided by the case of 'search' behaviours. This is not to say that such expectations require such explicit behavioural manifestations.
} 
the previously experienced whole - the apparent face of a loved one, as experienced on the basis of tacit expectations - and the reality. More generally, when peering into a crowd, although it may seem, in one sense, to be a richly detailed experience, again we can attend to sense in which the sensory detail is lacking. It is the fact that we usually simply attend to the 'whole', without paying close attention the limitations of our sensory perspective, that allows for this variety of misperception.

While there are undoubtedly alternative explanations of such misperceptions, the sensorimotor approach is well equipped to explain these experiences in a way that captures their phenomenology. As Noë says of the more general experience of seeing people in a crowd, 'Of course, it is not the case that you actually see everyone in sharp focus and uniform detail from the centre out to the periphery. We know that is not true. It does not seem as if it is true' (2009, p. 472). Likewise, in the case at hand, the experience of the face in the crowd does not present itself as being one in which one attends to the details of the face but experiences these details non-veridically. Rather, the experience seems to be both more momentary and more nebulous than would be suggested by such an explanation. When one does attend to the details of the face, the misperception is eliminated, which coheres with the claim that the error lies in the way the scene - and the face of the person, more specifically - is perceived without immediate sensory awareness of the details.

As discussed, sensorimotor expectations are activated against the backdrop of other expectations and judgements (e.g., Hurley \& Noë, 2006, p. 17), and they are also likely to be influenced by one's relations to others, desires, thought patterns and current emotional state (see also Sect. 5 below). A loved one can be deeply entrenched in one's world and their absence can extremely destabilising, and within such a context, sensorimotor expectations relating to the presence of the deceased are likely. As noted above, the non-veridical experience of seeing the deceased will also give rise to different affordances. The bereaved may be momentarily primed to approach the person, speak to them, etc., which will likely clash in a jarring way with one's explicit knowledge of the loved one's death. Through more protracted engagement with the world, one's perceptual experience ceases to suggest that the face is that of the deceased, which may give way to painful feelings of absence rather than presence.

Other non-veridical experiences associated with bereavement do not appear to be misperceptions of this sort and may instead be classified as full-blown hallucinations. It is to such experiences that we now turn.

\section{2 'Appearances' without reality and bereavement hallucinations}

Not all post-bereavement non-veridical experiences can be attributed to an atypical interpretation of a stimulus. Some subjects describe hallucinatory experiences that do not seem to map onto anything that is really there in their environment. These might be explained through the experience of non-veridical 'appearances' (associated with one's immediate sensory perspective), which are not integrated within the usual patterns of sensorimotor dependency. This may account for unusual sensory appearances that can occur while wearing vision disrupting goggles. Such 
experiences may also occur as a result of the sensorimotor disruption characteristic of bereavement. Although the sensorimotor account says little about why one might experience hallucinatory appearances in the first place, ${ }^{9}$ appealing to the notion of sensorimotor expectations does allow for an explanation of the distinctive character of a puzzling subset of non-veridical bereavement experiences. In particular, it can tell us why such experiences often lack 'perceptual presence'.

Understanding the perception of objects and scenes in terms of the sensory effects of one's own movement allows for a distinction between experiences of mind-independent whole objects and transient sensory changes or 'appearances' that occur as we move (e.g., see Noë, 2004, Chapter 3). As highlighted above, at least some of the time we can attend to our changing sensory perspectives, giving rise to a kind of duality to perceptual experience. One might notice, for example, the elliptical appearance of a plate when viewed from an angle or the fact that the volume of music appears quieter when the radio is further away. The sensorimotor approach can also thus allow for instances in which we experience (non-veridical) appearances, which are not situated within the usual patterns of sensorimotor contingency, making them lack objective import. Noë points out that experiences can be nonveridical along either the 'perspectival' (i.e., relating to one's sensory perspective) or 'factual' dimensions (relating to the 'gestalt' or whole). Atypical amodal completion allows for the latter type of experience, but other atypical experiences conform to the former, relating to appearances that are not properly integrated within one's sensorimotor attunement to the world. Where appearances are non-veridical, the perceiver may experience rogue sensations that lack the 'perceptual presence' characteristic of objective perception.

An example of this kind of experience is an after-image. After-images are experienced as overlaid on the visual field rather than as whole, perceptually present objects. These images appear to the agent in whatever direction they look, rather than allowing for changing sensory inputs as one engages with the world. ${ }^{10}$ Fuchs endorses a similar idea with regards hallucinatory experiences, stating:

$[F]$ rom an enactive point of view, hallucinations are only pseudo-perceptions which lack the sensorimotor cycles necessary for realistic perceiving on the first level. They may thus be regarded as products of the prefiguring activity of sensory or other brain systems which are projected into the field of awareness without resulting in sensorimotor interactions or perspectival change (this

\footnotetext{
9 See Seth (2014) for discussion of the possible mechanistic underpinnings of these kinds of experiences. He suggests synaesthesia (and plausibly the types of hallucinations under discussion) are explicable within a predictive processing model of the sensorimotor approach in terms of the brain's predictive model lacking counterfactual richness.

10 Relatedly, Ward (2012) uses such an approach to highlight why synaesthetic colour experiences do not adapt away as one might expect. With this approach, synaesthetic colour experiences are mere colour 'appearances', detached from the usual systematic sensorimotor patterns through which objective colours are experienced. Sensorimotor understanding regarding such regularities is not exercised in the synaesthesia case, and thus the experience does not present itself as being of 'real' colours. This is vindicated by the fact that synaesthetic colour experiences do not - as one may expect - get in the way of one's ability to perceive the actual colours of objects.
} 
is why they are frequently experienced by the patients as "not really perceptions"). In other words, hallucinations are the result of a decoupling of brain activity and normal body-environment feedback. (2020, p. 66)

Hallucinations, with this approach, often lack the perceptual presence fostered through one's grasp of how movement will induce perspectival changes. ${ }^{11}$ Such an approach could help to explain the nature of phantom smells, sights and sounds that occur following bereavement that cannot be attributed to any external stimulus. In such cases, we should expect the experience not to present itself as a part of an integrated scene in the way that the amodal completion cases above do. At least some reported bereavement hallucinations have the hallmarks of this kind of experience, and such experiences may seem especially puzzling. Consider the following case described by Schneck (1989), as cited by Sacks (2012):

A reporter brought the unexpected news one morning and Mitchell, greatly shaken, went up to tell his wife. On the way back downstairs he had an odd experience: he could see the face of Brooks, larger than life, smiling, and very distinct, yet looking as if it were made of dewy gossamer. When he looked down, the vision disappeared, but for ten days he could see it a little above his head to the left. (p. 136)

The described hallucinated face in this example would not allow for sensorimotor investigation because it lingers at the same point in the visual field no matter how the perceiver moves. The hallucinated appearance more closely resembles an afterimage than a genuine object, lacking perceptual presence and appearing like 'dewy gossamer' rather than an integrated aspect of the environment. Paying attention to the role of sensorimotor expectations allows for an explanation of this strange type of bereavement hallucination.

\subsection{Alterations to perceived affordances and quasi-sensory experiences of presence}

As discussed, the ways perception and action are entwined also encompass the fact that perception is generally action oriented. The sensorimotor approach draws upon the notion of 'affordances' - perceptible opportunities for action - from Gibson's ecological theory of perception (1979). Noë suggests that all objects of perception are, in some sense, affordances because all perception requires an attunement to the ways that our own actions can bring other parts of the world into view (2004, p. $106 ; 2015)$. He argues, 'What we are given, always, is an opportunity or affordance for further effort, engagement, negotiation, and skilful transaction' (2015, p. 14; see also Roberts, 2009). Beyond the sensorimotor profiles of objects, we also perceive them as affording a suite of larger scale intentional actions. We not only perceive the tomato as a whole, voluminous object, but also as an object that affords eating.

\footnotetext{
11 I shall not here address the question of whether one can ever have a hallucinatory experience that fully resembles veridical object perception, involving a sense of perceptual presence.
} 
As Di Paolo (2016, p. 230) points out, 'I don't normally inspect a bottle with my fingers to perceive in it a Gestalt shape as opposed to disjointed sensations; I pour some beer out of it. I don't regard the red tomato and consider its unseen sides and its voluminous presence; I cut it and add it to the salad'. The opportunities for action presented by the tomato structure our experience of it, and the action-oriented nature of perception allows us to competently interact with the environment.

Grief alters the opportunities for action that one experiences, and this is especially helpful in explaining quasi-sensory experiences of presence, which may involve no particular sense modality. At times, non-veridical bereavement experiences may be understood not in terms of atypical perceptual organisation (and thus is not to be explained in terms of atypical amodal completion or the experience of sensory appearances that are not properly integrated into the usual patterns of sensorimotor continency to which one is attuned) but rather in terms of atypical perception of affordances. For example, if one perceives the possessions of the deceased as affording possibilities for action, like laying them out for the deceased (as though she was still there to make use of them), or perceives a grave as affording the opportunity to converse, then the bereaved may report an experience of the presence of the deceased. It is important to note also that these alterations to experienced affordances can vary wildly depending upon the culture within which one is situated. For example, in Hong Kong it is common for the bereaved to report experiences of their loved one returning the form of an insect, allowing them the opportunity to speak to the deceased once more (Chan, et al., 2005). Drawing upon the notion of affordances, we can take the insect to afford conversing and respectful interaction, rather than, say, swatting away.

Ratcliffe (2020b) provides a detailed account of how alterations to the possibilities we experience in our environments can give rise to the some of the unusual experiences characteristic of grief. He highlights how these possibilities may result in an experience of the presence of the deceased:

Consider how one's home might be experienced in ways that implicate a partner: the sofa appears as something for us to sit on; these are the films we enjoy watching together; this is our bed. The suggestion is that, when the partner dies, a habitual world may remain largely intact for a time, despite an explicit, propositional recognition of the death. Things still appear as they did before and therefore imply the partner's potential or actual presence, in a diffuse, nonlocalized way that cannot be attributed to a more specific inventory of perceived properties. (p. 6)

Ratcliffe states that these possibilities may not be perceptual in nature. However, it does seem that at least some such possibilities can be construed in terms of the affordances offered by the perceptual landscape, thus allowing for further way of understanding grief's impact on perceptual experience. Grief results in alterations to the affordances that are salient to us as perceivers. Objects which previously gave rise to certain opportunities for intersubjective engagement, or which are strongly associated with the deceased, may no longer solicit the kinds of actions that they once did or conversely may continue to afford actions in ways that implicate the partner's presence (see also Sect. 5). 
One may find while grieving that activities lack their usual motivational appeal, where one feels numb emotionally (Roberts, 2019), or the world appears strangely lacking in opportunity (Ratcliffe, 2020b). According to Lindemann's (1963) research into acute grief, one may go through the motions of their daily routines, 'but these activities do not proceed in the automatic, self-sustaining fashion which characterizes normal work but have to be carried on with effort, as though each fragment of the activity became a special task. The bereaved is surprised to find how large a part of his customary activity was done in some meaningful relationship to the deceased and has now lost its significance' (p. 10). Our perceptions of the world are action-oriented and the possibilities for action that we experience are altered by bereavement. As we shall see in greater detail in the next section, consideration of affordances and how they relate to emotion and intersubjective relations with others, can serve to plug certain important omissions in the sensorimotor approach.

\section{Limitations of the sensorimotor approach}

We have seen that the sensorimotor approach has the resources to provide a plausible account of various puzzling types of post-bereavement perceptual phenomenology. However, grief is more bewildering, world-shattering, and painful than would be suggested by the comparison with other types of sensorimotor disruption, such as that induced by vision reversing goggles.

As it stands, the central resources of the sensorimotor approach do not provide a fleshed out explanation of why the loss of a significant person, but not the loss of an object like a car or a computer, should so frequently induce the kinds of anomalous experienced discussed in the preceding sections. Nor does it highlight other important differences between the sensorimotor disruption caused by bereavement and that exemplified by vision reversing goggles or by mundane cases of violated sensorimotor expectations, such as those caused by one's office being reorganised in one's absence. Part of the reason that anomalous post-bereavement perceptual experiences seem so importantly different from experiences involving more mundane violated sensorimotor expectations is that perceptual experience is closely intertwined with affect and intersubjectivity. The sensorimotor approach has been criticised in detail elsewhere for its silence regarding these aspects of experience (e.g., Bower \& Gallagher, 2013; Gallagher \& Bower, 2014), and non-veridical bereavement experiences provide a further illuminating case where perceptual phenomenology (the target explanandum of the sensorimotor theory) cannot be wholly divorced from the affective and the intersubjective. In this section, I provide an initial sketch of some natural avenues through which the sensorimotor approach can be supplemented to provide a richer account of post-bereavement perceptual phenomenology.

The shattering of expectations regarding the presence of a loved one is much more emotionally charged than most other instances of sensorimotor disruption. Although the sensorimotor approach does not focus upon affect and emotion, bereavement's emotional significance does have clear implications from a sensorimotor perspective. In particular, one's affective state is tied both to how one in fact interacts with the world, and the counterfactual opportunities for action one 
perceives as available to them. As one example, Lindemann $(1963$, p. 9) notes that when grief-stricken, one is likely to feel weak and fatigued, so the slightest effort is exhausting. Grief is then likely to have a profound effect on one's embodied interaction with the world; one's perceptual engagement itself become slower and feel more effortful than usual. A bereaved subject's perceptual experience may be coloured by how laborious it feels to read words on a page or to eat some food. Even the scope of one's attention is influenced by affective valence (Derryberry \& Tucker, 1994). The opportunities for action that are experienced as salient - and become solicitations (Rietveld \& Kiverstein, 2014) - depend upon one's concerns, emotions and one's relations with others. For a grieving subject, opportunities that would normally be enticing (eating a piece of chocolate cake, gazing at a beautiful sunset, etc.) may no longer have the same affective appeal, particularly where those activities would have previously been shared with the person who died. ${ }^{12}$ As Maclaren puts it, 'In grief like this, things within one's perceptual field no longer clearly call to one to do this or to say that' $(2011$, p. 62). As discussed in Sect. 4.3, on the flip side of this, other opportunities that would not normally solicit action may be experienced as salient while grieving; a grave or an insect might solicit conversation, when ordinarily they would not.

A further way in which bereavement's disruption is distinctive is that, as researchers have highlighted elsewhere, the intelligibility of one's projects, activities and routines can hinge upon another person so that when that person dies, these ways of engaging with the world cease to make sense altogether (e.g., Maclaren, 2011; Ratcliffe, 2020a). ${ }^{13}$ For example, as Ratcliffe (2020a, p. 659) highlights, one's activities and projects may be for the other person, thus ceasing to be comprehensible if that person dies. Ratcliffe notes that they may also be for 'us' and relate to things 'we' do together, and this 'we' may be irreducible to a 'you' and an 'I', so these activities likewise become unintelligible when the other person has died (ibid.). Maclaren relatedly says that for a person who is grieving the loss of someone central to their world, 'Not only his big life projects, but even his everyday routines had her as a condition of their meaningfulness' (p. 60). With such an approach, the loss of a person can thereby impair one's ability to meaningfully interact with the world in a way that cases of thwarted expectations due to a reorganised office does not. This goes some way towards explaining why anomalous post-bereavement phenomenology is a frequent occurrence, while other changes and absences rarely have such a striking impact upon our sensorimotor skills. Our ability to interact with the world in the meaningful and fluent ways can depend in important respects upon our relations with others, and thereby may be impaired through a bereavement. ${ }^{14}$

\footnotetext{
12 See De Haan, et al. (2013) for a helpful characterisation of the affective allure of affordances as a key dimension of the 'field' of affordances - 'the relevant possibilities for action that a particular individual is responsive to in a concrete situation' (p. 7, italics in original).

13 See also Guenther's (2013) account of how the devastating effects of social isolation expose how intercorporeal experiences of others are crucial in the sustenance of our ability to perceive the world as meaningful.

14 Another way in which a specific person's absence might profoundly disrupt one's sensorimotor engagement is through the dissolution of joint navigation of the world. Where a loved one dies, any collective worldly engagement with them ceases. Chemero (2016) introduces the notion of 'sensorimo-
} 
Relatedly, bereavement can impact the opportunities presented to us for regulating our own affective lives (Krueger \& Colombetti, 2018). A favourite song or relaxing bath might afford opportunities for altering and improving one's emotional state, for example, and likewise other people also present opportunities for shaping our emotional lives. For example, when faced with a threat, holding one's partner's hand can attenuate the activation of the neural systems involved in threat responses (Coan et al., 2006). Through a bereavement, a person that may have played a key part in regulating our emotional lives is no longer available (e.g., see Ratcliffe \& Byrne, 2021). This can make violated expectations relating the presence of the loved one especially disorienting, again shaping one's interactions with the world, and potentially further impairing one's sensorimotor skills.

This section has highlighted some avenues for supplementing the sensorimotor approach, so that it can tell a more fleshed out story post-bereavement perceptual phenomenology. Any sensorimotor disruption characteristic of grief will involve a crucial emotional component and bereavement may violently disrupt one's ability to engage in meaningful worldly interaction due to the ways in which this ability can hinge upon one's relations with others. A full account of the non-veridical experiences that can follow bereavement would require a more in-depth exploration of the role of intersubjective and affective factors in perceptual experience, so the discussion here should be read as simply one key building block towards such an account.

\section{Adapting to a new world}

This paper has argued that consideration of sensorimotor expectations and the action-oriented nature of perception allows for a phenomenologically accurate (although in certain respects, incomplete) account of post-bereavement non-veridical experiences. One's sensorimotor expectations are thwarted by the permanent absence of a loved one and perceived affordances are altered, which helps to explain the three key types of post-bereavement non-veridical experience. With other kinds of sensorimotor disruption, however, such as reversing goggles and sensory substitution, there is usually a comparatively speedy adaptation to the new patterns of sensorimotor contingency, occurring over several weeks of use (e.g., Kohler, 1962). Bereavement hallucinations and experiences of presence, on the other hand, seem to be more persistent. This is likely partly due to the crucial role played by our

\footnotetext{
Footnote 14 (continued)

tor empathy' - a kind of skilful perceptual and motor coordination with objects and with other people through which 'your lived body expands, and temporarily includes aspects of the non-bodily environment, whether they are tools or other humans' (p. 145). Just as a blind person might form a synergy with their cane, which allows them to perceive the world at the cane's tip rather than the cane in their hand, one might also form a system with another person through collective worldly engagement. In such cases, Chemero argues, people can (temporarily) form a coordinated and unified system. Such synergies seem especially plausible among, say, partners who share their lives. If this is the case, bereavement may result in the added disruption of breaking apart a unified system comprising the partners, further hindering one's sensorimotor abilities. Although further discussion of this aspect of sensorimotor impairment and bereavement is beyond the scope of the current paper, it presents an avenue for further research.
} 
intersubjective relations with others in our meaningful worldly interactions, as outlined in the previous section.

Recall that, as noted in Sect. 2, Grimby (1993) found that post-bereavement 'hallucinations' among the elderly decline significantly over the first year of the bereavement, which would be indicative of adaptation to our sensorimotor expectations, while experiences of the presence of the deceased did not. Others note that people continue to report sense of presence experiences may years after the loss (e.g., Steffen \& Coyle, 2011). This may suggest that while certain sensorimotor expectations regarding the dead are likely to adapt over the first year of bereavement, others can persist much longer. Adaptation to sensorimotor contingencies occurs in stages and may only be partial. ${ }^{15}$

This may have implications for frameworks for understanding grief. The continuing bonds approach to bereavement tell us that rather than grief being characterised by the severing of one's relationship with their loved one, it instead involves adaptation of this relationship. Recent work has emphasised post-bereavement non-veridical experiences as manifestations of one's 'continuing bonds' with the deceased (e.g., Klass et al., 1996; Steffen \& Coyle, 2011, 2017). Køster (2020) argues that the continuation of bonds with the dead relies upon an 'adequately sedimented' sense of the deceased in one's bodily memory. The sensorimotor theorist can endorse such a claim, but it is worth adding that, since sensorimotor adaptation can occur in stages at different rates, there may be several different types of connection we retain with the dead. Some continued connections are short-lived, and others can persist indefinitely. One type of continued relationship with the deceased can be understood in terms of one's continued sensorimotor expectations regarding the deceased, inducing non-veridical experiences. Atypical amodal completion, for example, seems to occur most frequently in the acute stages of grief. After those sensorimotor expectations have adapted to the absence of the dead, however, the altered perception of affordances, which may implicate the deceased in some non- or quasi-sensory manner (see Ratcliffe, 2020b), may persist. Other types of worldly engagement may involve the development of new relationships, perhaps with the memories and a (re) constructed image of the deceased, which might constitute further varieties of continuing bonds.

There is generally a gradual adaptation to loss in bereavement. As Parkes (1988, p. 56) says of spousal bereavement, 'Habits of action [...] and thought [...] must be revised if the survivor is to live as a widow'. Attig, who argues that grief can be understood a process of relearning the world, states, "[F]inding our way in the world after bereavement, that is, relearning the world, is not a matter of learning information about the world but learning how to be and act in the world differently in the light of our loss" (p. 107). We eventually learn how to fluently act in the world again. But grieving is a protracted process because, 'When we grieve, we must relearn virtually every object, place, event, relationships with others, and aspects of ourselves

\footnotetext{
15 In discussion of vision inverting goggles, O'Regan \& Noë note that adaptation can occur in stages: bodily skills like grasping objects may be re-mastered and these objects may appear 'normal', while writing might still appear reversed and unreadable (2001, p. 953).
} 
that the lives of those who have died have touched' (p. 122). Thus, fully adapting to the new patterns of sensorimotor dependency following a bereavement is likely to be a slow and difficult process.

Consideration of sensorimotor expectations and the circumstances under which these expectations go awry may allow for research into further types of atypical and non-veridical perceptual phenomena. The approach set out here may thus have applicability beyond the exploration of post-bereavement experiences. For now, though, it suffices to note that the sensorimotor account can capture and elucidate some of the most confusing and under-researched elements of bereavement phenomenology, which is, I hope, indicative of the approach's explanatory value.

Acknowledgements I am grateful to Matthew Ratcliffe, Louise Richardson, Emily Hughes, Eleanor Byrne, Jonny Lee, Pilar Lopez-Cantero, and Mara Neijzen for fruitful discussions and helpful comments. I also extend my gratitude to two anonymous reviewers, whose detailed feedback and suggestions helped me to greatly improve this paper. I thank the Arts and Humanities Research Council for supporting this research (grant ref. AH/T000066/1).

Funding This paper was written as part of the AHRC-funded project, 'Grief: A Study of Human Emotional Experience'.

Availability of data and material Not applicable.

Code availability Not applicable.

\section{Declarations}

Conflicts of interest/Competing interests Not applicable.

Open Access This article is licensed under a Creative Commons Attribution 4.0 International License, which permits use, sharing, adaptation, distribution and reproduction in any medium or format, as long as you give appropriate credit to the original author(s) and the source, provide a link to the Creative Commons licence, and indicate if changes were made. The images or other third party material in this article are included in the article's Creative Commons licence, unless indicated otherwise in a credit line to the material. If material is not included in the article's Creative Commons licence and your intended use is not permitted by statutory regulation or exceeds the permitted use, you will need to obtain permission directly from the copyright holder. To view a copy of this licence, visit http://creativecommons.org/licen ses/by/4.0/.

\section{References}

Ataria, Y. (2015). Trauma from an enactive perspective: The collapse of the knowing-how structure. Adaptive Behavior, 23(3), 143-154.

Attig, T. (1996). How we grieve: Relearning the world. Oxford University Press.

Bower, M., \& Gallagher, S. (2013). Bodily affects as prenoetic elements in enactive perception. Phenomenology and Mind, 4(1), 78-93.

Bowlby, J. (1980). Attachment and loss, vol 3: Loss, sadness, and depession. Hogarth Press.

Castelnovo, A., Cavallotti, S., Gambini, O., \& Agostino, A. D. (2015). Post-bereavement hallucinatory experiences : A critical overview of population and clinical studies. Journal of Affective Disorders, $186,266-274$.

Chan, C. L., et al. (2005). The experience of Chinese bereaved persons: A preliminary study of meaning making and continuing bonds. Death Studies, 29, 923-947.

Chemero, A. (2016). Sensorimotor empathy. Journal of Consciousness Studies, 23(5-6), 138-152. 
Clayton, P. J. (2000). Bereavement. In G. Fink (Ed.), Encyclopedia of stress (1st ed., pp. 304-311). Academic Press.

Coan, J. A., Schaefer, H. S., \& Davidson, R. J. (2006). Lending a hand: Social regulation of the neural response to threat. Psychological Science, 17(12), 1032-1039.

Degenaar, J. (2013). Through the inverting glass: First-person observations on spatial vision and imagery. Phenomenology and the Cognitive Sciences, 13(2), 373-393.

de Haan, S., Rietveld, E., Stokhof, M., \& Denys, D. (2013). The phenomenology of deep brain stimulation-induced changes in OCD: An enactive affordance-based model. Frontiers in Human Neuroscience, 7(653), 1-14.

Derryberry, D., \& Tucker, D. M. (1994). Motivating the focus of attention. In P. M. Niedenthal \& S. Kitayama (Eds.), The Heart's Eye: Emotional influences in perception and attention (pp. 167-196). Academic Press.

Di Paolo, E. (2016). Participatory object perception. Journal of Conciousness Studies, 5-6, 228-258.

Field, N. P., \& Filanosky, C. (2009). Continuing Bonds, Risk Factors for Complicated Grief, and Adjustment to Bereavement. Death Studies, 34(1), 1-29.

Fuchs, T. (2018). Presence in absence. The ambiguous phenomenology of grief. Phenomenology and the Cognitive Sciences, 17(1), 43-63.

Fuchs, T. (2020). Delusion, Reality, and Intersubjectivity: A Phenomenological and Enactive Analysis. Philosophy, Psychiatry, \& Psychology, 27(1), 61-79.

Gallagher, S. A., \& Bower, M. (2014). Making enactivism even more embodied. Avant, 5(2), 232-247.

Gibson, J. J. (1979). The ecological approach to visual perception. Houghton Mifflin.

Goldie, P. (2011). Grief: a narrative account. Ratio, XXIV(2), 119-137.

Grimby, A. (1993). Bereavement among elderly people: Grief reactions, post-bereavement hallucinations and quality of life. Acta Psychiatrica Scandinavica, 87(1), 72-80.

Guenther, L. (2013). Solitary confinement: Social death and its afterlives. University of Minnesota Press.

Hofer, M. (1984). Relationships as regulators: A Psychobiologic perspective on bereavement. Psychosomatic Medicine, 46(3), 183-198.

Hurley, S. (1998). Consciousness in action. Harvard University Press.

Hurley, S., \& Noë, A. (2003). Neural plasticity and consciousness. Biology and Philosophy, 18(1), $131-168$.

Hurley, S., \& Noë, A. (2006). Can hunter-gatherers hear color? In G. Brennan, R. Goodin, \& M. Smith (Eds.), Common minds essays in honour of Philip Pettit. Oxford University Press.

Kirchhoff, M., \& Kiverstein, J. (2019). Extended consciousness and predictive processing: A third-wave view. Routledge.

Klass, D., Silverman, P. R., \& Nickman, S. L. (1996). Continuing bonds: New understandings of grief. Routledge.

Kohler, I. (1962). Experiments with goggles. Scientific American, 206, 62-72.

Kohler, I. (1964). The formation and transformation of the perceptual world. Psychological Issues, 3(4), $1-173$

Køster, A. (2020). Longing for concreteness: how body memory matters to continuing bonds. Mortality, 25(4), 389-401

Køster, A. (2021). The felt sense of the other: contours of a sensorium. Phenomenology and the Cognitive Sciences, 20, 57-73

Krueger, J., \& Colombetti, G. (2018). Affective affordances and psychopathology. Discipline Filosofiche, 2(18), 221-247.

Lindemann, E. (1963). Symptomatology and management of acute grief. Pastoral Psychology, 14, 8-18.

Maclaren, K. (2011). Emotional clichés and authentic passions: A phenomenological revision of a cognitive theory of emotion. Phenomenology and the Cognitive Sciences, 10, 45-65.

Myin, E., O'Regan, J. K., \& Myin-Germeys, I. (2015). From a sensorimotor account of perception to an interactive approach to psychopathology. In R. Gennaro (Ed.), Disturbed consciousness: New essays on psychopathology and theories of consciousness (pp. 347-368). MIT Press.

Noë, A. (2002). Is the visual world a grand illusion? Journal of Consciousness Studies, 9(5-6), 1-12.

Noë, A. (2004). Action in perception. MIT Press.

Noë, A. (2006). Experience of the world in time. Analysis, 66(289), 26-32.

Noë, A. (2009). Conscious reference. The Philosophical Quarterly, 59(236), 470-482.

O'Regan, J. K., \& Noë, A. (2001). A sensorimotor account of vision and visual consciousness. Behavioral and Brain Sciences, 24(5), 939-1031. 
Parkes, C. M. (1970). "Seeking" and "finding" a lost object: Evidence from recent studies of the reaction to bereavement. Social Science \& Medicine, 4(2), 187-201.

Parkes, C. M. (1988). Bereavement as a psychosocial transition: Process of adaptation to change. Journal of Social Issues, 44, 53-65.

Parkes, C. M. (1996). Bereavement: Studies of grief in adult life. Penguin Books.

Parkes, C. M., \& Prigerson, H. (2010). Bereavement: Studies of grief in adult life (4th ed.). Routledge.

Ratcliffe, M. (2017). Grief and the unity of emotion. Midwest Studies in Philosophy, XLI, 154-175.

Ratcliffe, M. (2020a). Towards a phenomenology of grief: Insights from Merleau-Ponty. European Journal of Philosophy, 28(3), 657-669

Ratcliffe, M. (2020b). Sensed presence without sensory qualities: a phenomenological study of bereavement hallucinations. Phenomenology and the Cognitive Sciences, 1-16

Ratcliffe, M., \& Byrne, E. (2021). The interpersonal and social dimensions of emotion regulation in grief. In A. Køster \& E. H. Kofod (Eds.), Grief experience: cultural, existential and phenomenological perspectives. Routledge.

Rees, W. D. (1971). The hallucinations of widowhood. British Medical Journal, 4, 37-41.

Rees, W. D. (1975). The bereaved and their hallucinations. In B. Schoenberg (Ed.), Bereavement its psychosocial aspects (pp. 66-71). Columbia University Press.

Rietveld, E., \& Kiverstein, J. (2014). A rich landscape of affordances. Ecological Psychology, 26, 325-352.

Roberts, T. (2009). Understanding 'sensorimotor understanding.' Phenomenology and the Cognitive Sciences, 9(1), 101-111.

Roberts, T. (2019). Feeling nothing: Numbness and emotional absence. European Journal of Philosophy, 27, 187-198.

Sacks, O. (2012). Hallucinations. Alfred A. Knopf

Schneck, J. M. S. (1989). Weir Mitchell's visual hallucinations as a grief reaction. American Journal of Psychiatry, 146(3), 409.

Seth, A. K. (2014). A predictive processing theory of sensorimotor contingencies: Explaining the puzzle of perceptual presence and its absence in synesthesia. Cognitive Neuroscience, 5(2), 97-118.

Steffen, E., \& Coyle, A. (2011). Sense of presence experiences and meaning-making in bereavement: A qualitative analysis. Death Studies, 35(7), 579-609.

Steffen, E., \& Coyle, A. (2017). "I thought they should know ... that daddy is not completely gone": A case study of experiences in bereavement and family meaning-making. OMEGA - Journal of Death and Dying, 74(4), 363-385.

Taylor, J. (1962). The behavioral basis of perception. Yale University Press.

Thompson, E. (2007). Mind in life: Biology, phenomenology, and the sciences of mind. Harvard University Press.

Varela, F., Thompson, E., \& Rosch, E. (1991). The embodied mind. MIT Press.

Ward, D. (2012). Why don't synaesthetic colours adapt away? Philosophical Studies, 159, 123-138.

Ward, D. (2016). Achieving transparency: An argument for enactivism. Philosophy and Phenomenological Research, 93(3), 650-680

Publisher's Note Springer Nature remains neutral with regard to jurisdictional claims in published maps and institutional affiliations. 